\title{
Sensibilidad aumentada a lidocaína: estudio genético de un caso
}

\author{
Daniela Bravo ${ }^{1}$, Antonello Penna ${ }^{1,2}$, Luis Michea ${ }^{3}$, Andrés Stutzin ${ }^{1,3}$
}

\begin{abstract}
Systemic toxicity by local anesthetics (LAs) is a severe and feared complication in anesthetic practice that generally results from the administration of an inappropriately high dose of LAs or an injection at an inappropriate place, either intravascular or a site with high absorption[1]. However, it is known that the susceptibility to these drugs may vary within the population, which may occur due to genetic changes in the LA binding site, located in the potentialdependent $\mathrm{Na}$ + channels $\left(\mathrm{N}_{\mathrm{av}}\right)$, thus increasing or decreasing its affinity and, therefore, its clinical consequences. We present a case of a 61 years-old female patient with a medical history of increased sensitivity to LAs. In this scenario, a genetic study was performed to exclude a $\mathrm{N}_{\mathrm{av}}$ channel dysfunction.
\end{abstract}

\section{RESUMEN}

La toxicidad sistémica por anestésicos locales (ALs) es una grave y temida complicación en la práctica anestésica que generalmente resulta de la administración de una dosis inapropiadamente alta de ALs o a una inyección en un lugar inadecuado, llámese intravascular o un sitio al alta absorción[1]. A pesar de lo anterior, es conocido que la susceptibilidad al efecto de estos fármacos puede variar dentro de la población, lo cual puede ocurrir debido a cambios genéticos en el sitio de unión de los $\mathrm{AL}$, localizado en los canales de $\mathrm{Na}+$ dependientes de potencial $\left(\mathrm{N}_{\mathrm{ay}}\right)$, incrementando o disminuyendo así su afinidad y, por ende, sus consecuencias clínicas. Presentamos el caso de una paciente de 61 años con historia de sensibilidad aumentada a Als. En este escenario, se le ofreció un estudio genético para excluir una disfunción específica a nivel de canal $\mathrm{N}_{\mathrm{av}}$

\section{Key words:}

Sodium channel, canalopathy, hypersensitivity to local anesthetic, toxicity by local anesthetic

Departamento de Anestesiología y Medicina Perioperatoria, Hospital Clínico Universidad de Chile.

Centro de Investigación Clínica Avanzada (CICA), Hospital Clínico Universidad de Chile.

Instituto de Ciencias Biomédicas (ICBM), Facultad de Medicina, Universidad de Chile.

Fecha de recepción: 25 de noviembre de 2019

Fecha de aceptación: 26 de noviembre de 2019

\section{ORCID}

https://orcid.org/0000-0003-0611-3623

Correspondencia:

Dra. Daniela Bravo

Email: danibravoadvis@gmail.com 


\section{Introducción}

L a toxicidad sistémica por anestésicos locales (ALs) es una grave y temida complicación en la práctica anestésica que generalmente resulta de la administración de una dosis inapropiadamente alta de ALs o a una inyección en un lugar inadecuado, llámese intravascular o un sitio al alta absorción[1]. A pesar de lo anterior, es conocido que la susceptibilidad al efecto de estos fármacos puede variar dentro de la población, lo cual puede ocurrir debido a cambios genéticos en el sitio de unión de los $\mathrm{AL}$, localizado en los canales de $\mathrm{Na}^{+}$dependientes de potencial $\left(\mathrm{N}_{\mathrm{av}}\right)$, incrementando o disminuyendo así su afinidad $\mathrm{y}$, por ende, sus consecuencias clínicas.

\section{Caso clínico}

A.G.J.G., mujer de 61 años, con antecedente de hipertensión arterial y obesidad, ingresó a nuestro centro para una reparación artroscópica de una rotura de manguito rotador. Durante la evaluación preanestésica la paciente refirió ser alérgica a la lidocaína, lo que obligó a indagar detenidamente en este antecedente.

El episodio que determinó esta supuesta condición ocurrió al realizarse una resección de un quiste de una de sus muñecas bajo anestesia regional endovenosa, donde dice haber tenido una pérdida de conciencia abrupta al liberarse el manguito de isquemia. Lamentablemente, desconocía mayores detalles de este episodio, aunque negó haber requerido una estadía en cuidados intensivos. A este evento se suma el relato de malestar general, sensación de sabor amargo y visión exacerbada de colores durante una cesárea al recibir una anestesia peridural tras un trabajo de parto fracasado y, por último, que al recibir anestesia infiltrativa para procedimientos dentales siente palpitaciones, malestar general y sensación de calor en su cuerpo.

Estos antecedentes parecían, en el caso de la cirugía de muñeca, ser compatibles con manifestaciones neurológicas de una toxicidad sistémica ocurrida tras la liberación precoz del mango de isquemia o por una dosis elevada de lidocaína, en el caso de la cesárea explicables por malestares del contexto clínico, y en el caso de las anestesias dentales por absorción del vasoconstrictor.

Dada la carga de dolor de la intervención a la cual se sometería la paciente (donde el estándar es un bloqueo analgésico del plexo braquial) y a la poca consistencia de un cuadro de alergia real a ALs, tras la monitorización e instalación de una vía venosa periférica, se administró una dosis subterapéutica de lidocaína $(0,3 \mathrm{mg} / \mathrm{kg})$, ante lo cual la paciente rápidamente refirió palpitaciones, sabor amargo en la boca, sensación de lengua pesada y adormecida y malestar general. El evento fue breve y autolimitado, no demostró cambios electrocardiográficos y no hubo alteración del estado de conciencia.

En este escenario, decidimos brindarle una anestesia y analgesia libre de anestésicos locales, y ante la ausencia de una condición clínica que explicara esta probable mayor sensibilidad a los ALs, se le ofreció a la paciente la realización de un estudio genético (de secuenciación exómica para identificar y analizar las variantes genómicas) de sus subtipos de $\mathrm{N}_{\mathrm{av}}$.

\begin{tabular}{|c|c|c|c|c|}
\hline Canal & Transcrito & Cambio de codón & Impacto & 1.000 genomas \\
\hline Nav1.1 & Ala1067Thr & Gca/Aca & $\begin{array}{l}\text { No patológica } 8 \text { repor- } \\
\text { tes previos }\end{array}$ & G:21\% y $A: 79 \%$ \\
\hline Nav1.2 & Arg19Lys & $\mathrm{aGg} / \mathrm{aAg}$ & $\begin{array}{l}\text { No patológica } 3 \text { repor- } \\
\text { tes previos }\end{array}$ & G:92\% y $A: 8 \%$ \\
\hline Nav1.7 & Trp1150Arg & Tgg/Cgg & Eritromelalgia & $\mathrm{T}: 11 \%$ y $\mathrm{C}: 89 \%$ \\
\hline Nav1.8 & Met1713Val & Atg/Gtg & Benigna 1 reporte & A:0\% y $G: 100 \%$ \\
\hline Nav1.8 & Leu1092Pro & $\mathrm{cTa} / \mathrm{cCa}$ & Benigna sin reporte & $\mathrm{T}: 78 \%$ y $\mathrm{C}: 22 \%$ \\
\hline Nav1.8 & Val1073Ala & $\mathrm{gTc} / \mathrm{gCc}$ & Benigna 1 reporte & $\mathrm{T}: 24 \%$ y $\mathrm{C}: 76 \%$ \\
\hline Nav1.8 & Ile962Val & Att/Gtt & - & No descrita \\
\hline Nav1.8 & Ser509Pro & $\mathrm{Tcc} / \mathrm{Ccc}$ & Benigna sin reporte & $\mathrm{T}: 86 \%$ y $\mathrm{C}: 14 \%$ \\
\hline
\end{tabular}

Hallazgos del estudio genómico del caso clínico según subtipo de canal de $\mathrm{Na}+$ dependientes de potencial $\left(\mathrm{N}_{\mathrm{av}}\right)$. 


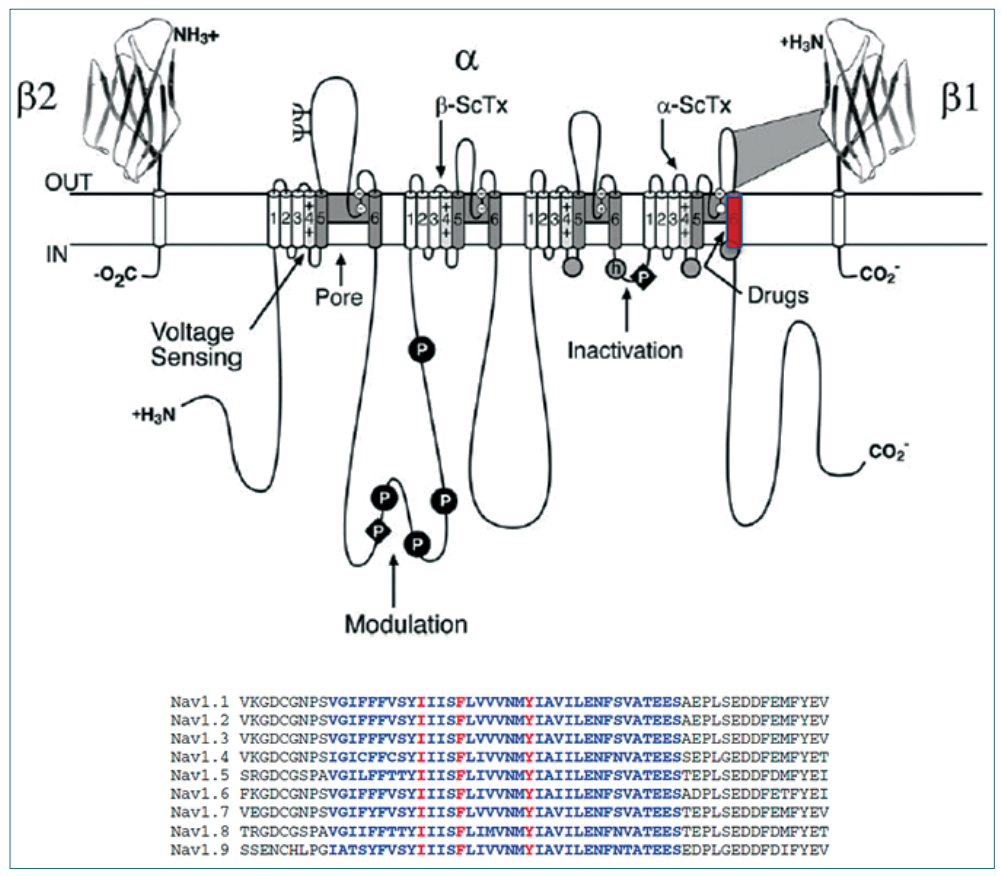

Figura 1. Esquema de la subunidad $\alpha$ de los canales de $\mathrm{Na}^{+}$dependientes de potencial $\left(N_{a y}\right)$ y secuencia de aminoácidos del sitio de unión a anestésicos locales encontrada de los distintos subtipos de $\mathrm{N}_{\text {av }}$ En rojo los tres aminoácidos clave para la interacción.

\section{Marco teórico}

Los ALs se caracterizan por interrumpir los potenciales de acción que se transmiten a través de las fibras aferentes y eferentes por medio de un bloqueo de los canales de sodio voltaje dependiente $\left(\mathrm{N}_{\mathrm{av}}\right)$. Los $\mathrm{N}_{\mathrm{av}}$ conforman una familia de 9 subtipos de canales iónicos $\left(N_{a v} 1,1\right.$ a $\left.N_{a v} 1,9\right)$, de los cuales sólo el $N_{a v} 1,4$ y $N_{a v} 1,5$ no se expresan en el sistema nervioso central y/o periférico. El $\mathrm{N}_{\mathrm{av}} 1,4$ se expresa en el músculo esquelético, mientras el $N_{a v} 1,5$ en el músculo cardíaco. Por lo tanto, el efecto anestésico de los ALs es secundario al bloqueo de los $N_{a v} 1,1$ a $N_{a v} 1,3 y / o$ $N_{a v} 1,6$ a $N_{a v} 1,9[2]$. Cambios genéticos en los $N_{a v^{\prime}}$ ya sean mutaciones o polimorfismos, dan cuenta de canalopatías como epilepsias, insensibilidad al dolor o arritmias, entre otras[2]. Por ejemplo, una mutación en el $\mathrm{N}_{\mathrm{av}} 1,7$ determina la expresión de un canal no funcional produciendo una insensibilidad al dolor de los sujetos que la poseen. Por otro lado, se ha descrito una mutación doble que incrementa la afinidad del $\mathrm{N}_{\mathrm{av}} 1,5$ con lidocaína, lo que explica una mayor susceptibilidad de cursar con una arritmia denominada síndrome de Brugada, si es que a un sujeto se le administra una dosis de ésta[2].

Por otra parte, la alergia por hipersensibilidad real (tipo IV) a los ALs es una entidad poco usual debido a que su molécula es demasiado pequeña para ser inmunogénicamente capaz de desencadenar este tipo de respuesta, sin embargo, ocasionalmente pueden constituir haptenos[3].

La lidocaína, al igual que los otros anestésicos locales, deben traspasar la membrana plasmática para bloquear el poro de los $N_{a v}$ por el lado intracelular, y este bloqueo ocurre preferencialmente cuando el canal se encuentra en un estado activo (abierto) e inactivo, por ello el bloqueo de los anestésicos locales se caracteriza por ser "uso dependiente" lo que significa que el AL bloquea a los $N_{\mathrm{av}}$ en la medida que estos se van activando[4]. El sitio de unión de los ALs se ha estudiado extensivamente, de hecho, se ha determinado que tres aminoácidos localizados en el dominio IV de los $\mathrm{N}_{\mathrm{av}}$ son claves para el bloqueo (Figura 1)[4]. Específicamente, estos aminoácidos se encuentran en la cara intracelular del poro del canal y mutaciones realizadas en estudios in vitro disminuyen o incrementan la afinidad del AL por su sitio de unión, lo que se expresa en un menor o en un mayor bloqueo de la actividad de los $\mathrm{N}_{\mathrm{av}}$, respectivamente[5].

\section{Metodología}

Con la aprobación del Comité de Ética del Hospital Clínico de la Universidad de Chile, se procedió a 
la obtención de una muestra de sangre periférica de nuestra paciente para así estudiar su ADN genómiCo, tras lo cual se realizó una secuenciación exómica completa (WES) por la compañía BGI Americas en la plataforma Illumina Hiseq. Se identificaron las variantes genómicas de 7 subtipos de interés de los $\mathrm{Na}_{\mathrm{v}}$ y se comparó la secuencia con la referencia genómica humana GRCh37/HG19. Luego, se estudió cada una de las variantes utilizando distintas bases de datos para determinar la relevancia de éstas.

\section{Resultados}

La secuenciación del genoma de la paciente fue de alta calidad, con un promedio de secuenciación de $99,7 \%$ del exoma y una profundidad media de 160,1 veces. Se identificaron 41.751 polimorfismos de nucleótido único (SNP) y, de estos, 9.272 fueron con cambio de sentido. En los 7 subtipos de Nav analizados se detectaron 40 SNPs, de los cuales 8 cambiaron el sentido 1 en el gen SCN1A $\left(N_{\text {av }} 1,1\right)$, 1 en el SCN2A $\left(N_{a v}, 2\right), 1$ en el SCN9A $\left(N_{a v} 1,7\right)$ y 5 en el SCN10A $\left(\mathrm{N}_{\mathrm{av}} 1,8\right)$. Posteriormente, al comparar estas variantes con la base de datos del Proyecto 1.000 genomas, se pudo determinar que 7 de las 8 variantes detectadas han sido descritas y tienen una prevalencia que va de un $8 \%$ a un $100 \%$, mientras una variante no había sido descrita. Finalmente, no se detectaron cambios relevantes en el sitio de unión de los ALs en los 7 subtipos de los $\mathrm{Na}_{\mathrm{v}}$.

\section{Discusión}

En nuestro Hospital detectamos este caso inusual de una mayor sensibilidad a la lidocaína, dado que con una dosis francamente subterapéutica $(0,3 \mathrm{mg} / \mathrm{kg})$ refirió sintomatología que habitualmente se presenta con dosis mayores y que serían mediados por los $\mathrm{N}_{\mathrm{av}}$ expresados en el sistema nervioso central y/o periférico.

La identificación de pacientes que presentan condiciones clínicas que escapan a la norma abre una puerta para conocer más a fondo mecanismos e interacciones relevantes que permiten ampliar el conocimiento y mejorar nuestro desempeño.

La relevancia de estudiar posibles cambios genéticos que pueden tener un efecto en la interacción de los ALs con los $\mathrm{N}_{\mathrm{av}}$, resulta clave tanto para determinar el riesgo que pueden tener los sujetos (y sus familias) que posean estos cambios genéticos, como también para comprender de mejor manera el mecanismo de acción de los ALs y los $\mathrm{N}_{\text {av }}$

En este caso puntual la no realización de un bloqueo analgésico significaba una merma significativa en la calidad del manejo del dolor postoperatorio de la paciente, por lo que se realizó esta prueba de lidocaína bajo condiciones controladas con el fin de tratar de aclarar su dudosa condición. Lamentablemente, no fue posible conocer la causal última de las manifestaciones de mayor sensibilidad a lidocaína. Sin embargo, el estudio realizado es sólo la etapa inicial de una indagatoria acabada que comprende el estudio de otros blancos y/o proteínas asociadas.

\section{Referencias}

1. 1. Neal JM, Barrington MJ, Fettiplace MR, Gitman M, Memtsoudis SG, Mörwald EE, et al. The third american society of regional anesthesia and pain medicine practice advisory on local anesthetic systemic toxicity. Reg Anesth Pain Med. 2018 Feb;43(2):113-23. https://doi.org/10.1097/ AAP.0000000000000720 PMID:29356773

2. England S, de Groot MJ.
Subtype-selective targeting of voltage-gated sodium channels. Br J Pharmacol. 2009 Nov;158(6):1413-25. https:// doi.org/10.1111/j.14765381.2009.00437.x PMID:19845672

3. Becker DE, Reed KL. Essentials of local anesthetic pharmacology. Anesth Prog. 2006;53(3):98-108. https://doi. org/10.2344/00033006(2006)53[98:EOLAP] 2.0.CO;2 PMID:17175824

4. Scholz A. Mechanisms of (local) anaesthetics on voltage-gated sodium and other ion channels. Br J Anaesth. 2002 Jul;89(1):5261. https://doi.org/10.1093/bja/ aef163 PMID:12173241

5. Ragsdale DS, McPhee JC, Scheuer T, Catterall WA. Molecular Determinants of State Dependent Block of $\mathrm{Na}$ Channels by Local Anesthetics. Science (80-) . 1994; 265 (5179):17241728. https://doi.org/10.1126/ science. 8085162 . 